Supplement to

\title{
Coupling of Retrograde Flow to Force Production During Malaria Parasite Migration
}

Katharina A. Quadt ${ }^{1, *}$, Martin Streichfuss ${ }^{1,2^{*}}$, Catherine A. Moreau ${ }^{1}$, Joachim P. Spatz $^{2}$ and Friedrich Frischknecht ${ }^{1, \#}$

Supporting Figure 1
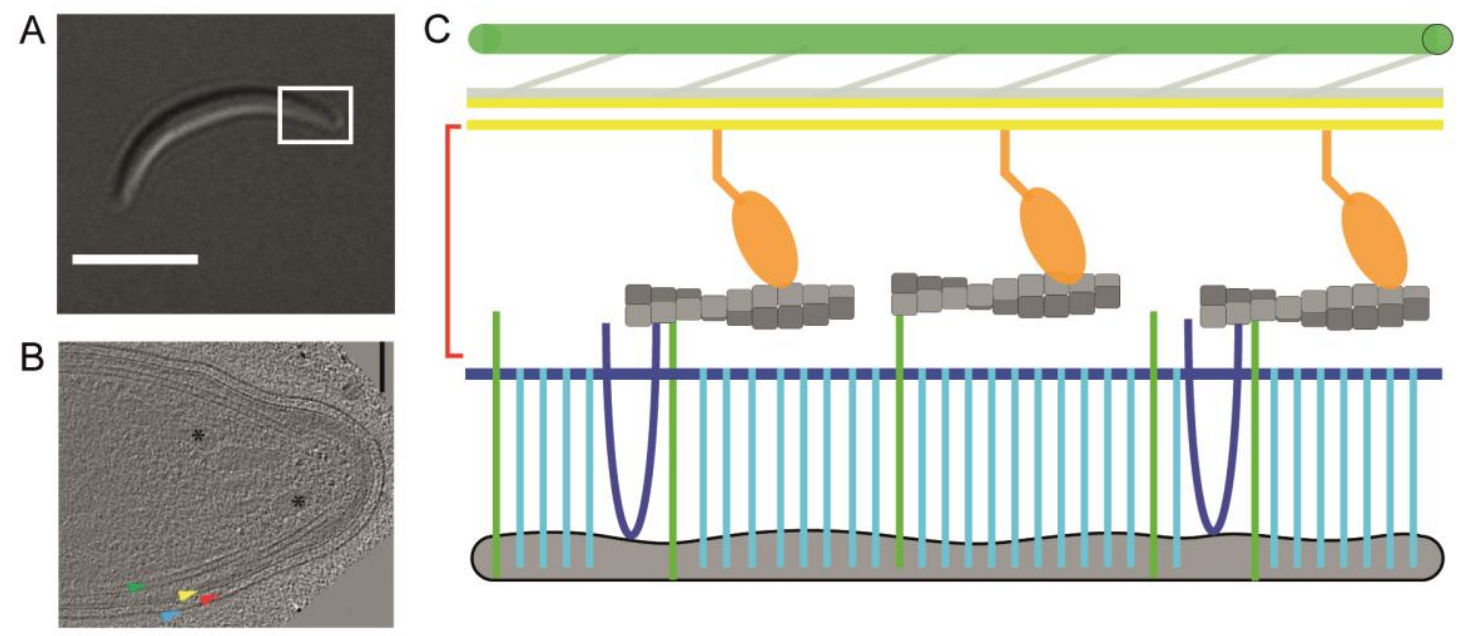

(A) DIC Image of a gliding sporozoite. Scale bar $5 \mu \mathrm{m}$. Box indicates area visualized by tomography in B. (B) Tomographic reconstruction of apical end taken from reference ${ }^{27}$. Arrowheads indicate structures as highlighted with the same colors in C. (C) Schematic model of parasite pellicle and gliding motor. Microtubules (green) are linked (gray) to the sub-pellicular network (gray) and inner membrane complex (yellow). Within the supra-alveolar space (red bracket), myosin A (orange) is anchored to the IMC and binds actin filaments (light and dark gray). Surface proteins at the plasma membrane (dark blue) include circumsporozoite protein (cyan), Thrombospondin-Related Anonymous Protein (light green), TRAP-like protein (blue), substrate (dark gray). 
Supporting Figure 2 in support of Figure 1D-F: tracking beads on hemolymph sporozoites.
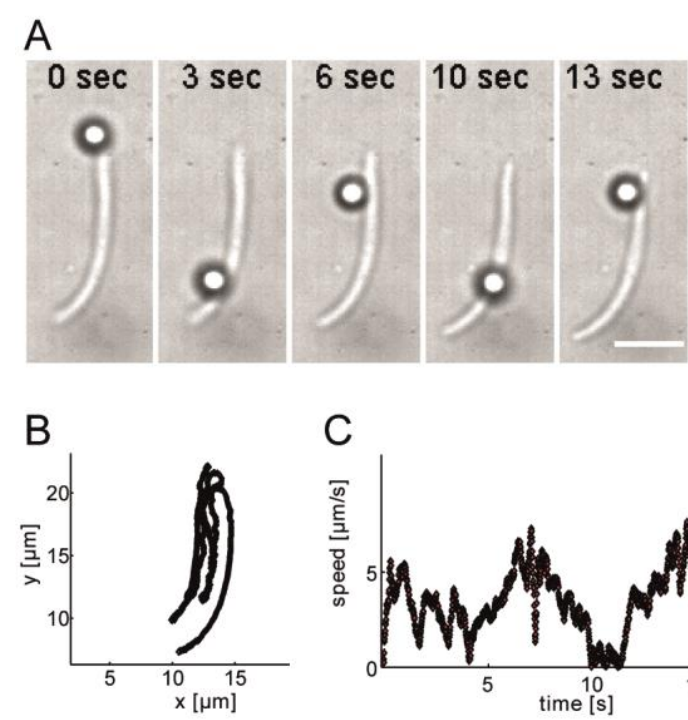

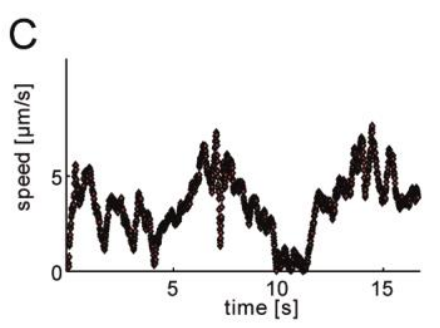

(A) Image series of patch-gliding hemolymph-sporozoite transporting a bead back and forth (see also Supporting Movie 2). (B) Trajectory plot of the transported bead. (C) Corresponding speed plots with peaks around 6-8 $\mu \mathrm{m} / \mathrm{s}$.

Supporting Figure 3 in support of Figure 5B: Force bars at $190 \mathrm{pN}$

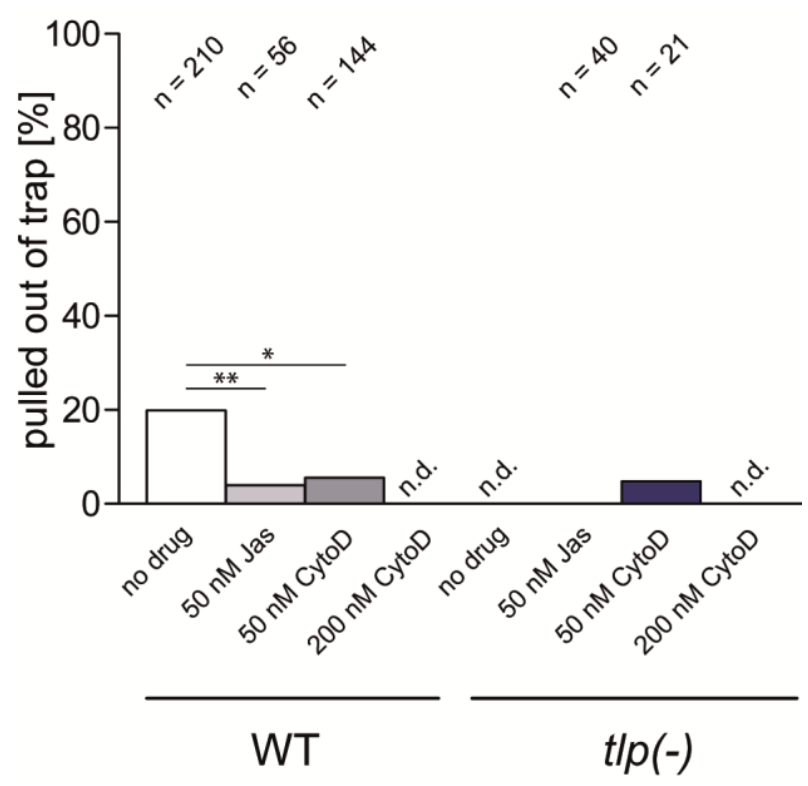

Supporting Movie 1 to support Figure 1A: WT sporozoite translocating bead rearwards. Movie recorded with 60 frames per second. 
Supporting Movie 2 to support Figure 1 D-F: WT sporozoite with a bead stuck at the rear end undergoing extreme stop-and-go gliding motility. Movie recorded with 500 frames per second.

Supporting Movie 3 to support Supporting Figure 2: WT hemolymph sporozoite translocating a bead in a back-and-forth manner. Movie recorded with 100 frames per second.

Supporting Movie 4 to support Figure 2C: WT sporozoite pulling bead out the trap.

Movie recorded with 100 frames per second.

Supporting Movie 5 to support Figure 3A: $t l p(-)$ sporozoite translocating bead. Movie recorded with 100 frames per second.

Supporting Table 1: Variance of data from force measurements on seven different days from wild type sporozoites probed at three different forces as illustrated in Figure 2E.

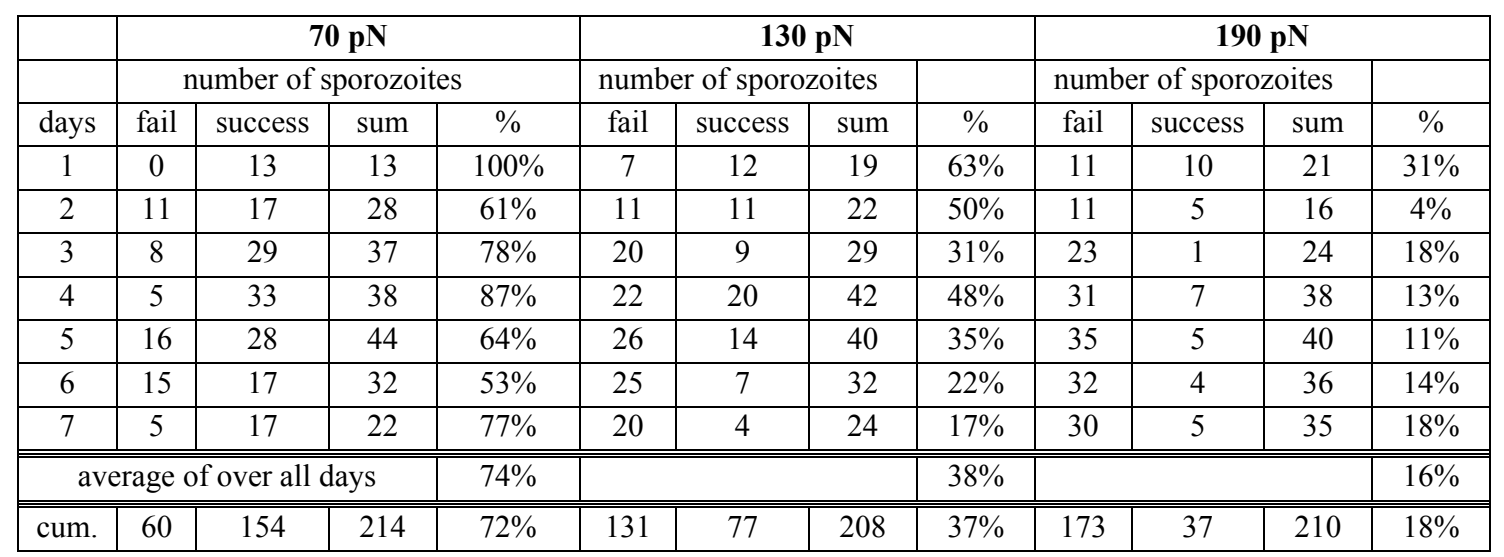


Experimental outcome was two-fold: a sporozoite failed or managed to pull the bead out of the trap, which was noted for every sporozoite and listed under 'fail' or 'success' respectively for the number of sporozoites examined at that particular day. 'sum' indicates the total of sporozoites examined at a particular day. '\%' indicates the percentage of sporozoites that were successfully pulled out from the trap. 'cum.' indicates the cumulative number of sporozoites that failed or succeeded to pull a bead out of a trap over all 7 days. Note the very similar outcomes for cumulative and averaged results, e.g. $74 \%$ vs $72 \%$ for $70 \mathrm{pN}, 38 \%$ vs $37 \%$ for $130 \mathrm{pN}$ and $16 \%$ vs $18 \%$ for $190 \mathrm{pN}$.

Supporting Table 2: Statistical analysis

\begin{tabular}{|l|l|c|c|c|c|c|}
\hline \multicolumn{2}{|c|}{} & \multicolumn{3}{|c|}{ Force } & Retrograde flow & Time until Transport \\
\hline & & $\mathbf{7 0} \mathbf{~ p N}$ & $\mathbf{1 3 0} \mathbf{~ p N}$ & $\mathbf{1 9 0} \mathbf{~ p N}$ & unpaired t-test & Mann-Whitney test \\
\hline WT & WT+50nM CytoD & $<0,0001$ & $<0,0001$ & 0,0006 & 0,4343 & 0,0003 \\
\hline WT & WT+50nM Jas & $<0,0001$ & $<0,0001$ & 0,0208 & $<0,0001$ & 0,5864 \\
\hline WT & WT+200nM CytoD & $<0,0001$ & & & $<0,0001$ & $<0,0001$ \\
\hline WT+50nM CytoD & WT+200nM CytoD & $<0,0001$ & & & $<0,0001$ & $<0,0001$ \\
\hline$t l p(-)$ & $t l p(-)+50 n M$ Jas & 0,1183 & 0,0668 & & 0,0159 & 0,084 \\
\hline$t l p(-)$ & $t l p(-)+50 n M$ CytoD & 0,0073 & 0,0023 & & 0,1603 & $<0,0001$ \\
\hline$t l p(-)$ & $t l p(-)+200 n M$ CytoD & 0,0024 & & & $<0,0001$ & $<0,0001$ \\
\hline$t l p(-)+50 n M$ CytoD & $t l p(-)+200 n M$ CytoD & $<0,0001$ & & & 0,0017 & $<0,0001$ \\
\hline WT & $t l p(-)$ & $<0,0001$ & $<0,0001$ & & $<0,0001$ & 0,5659 \\
\hline
\end{tabular}

*gray cells: not determined 